\title{
About gravitational interaction in astrophysics in Riemann-Cartan space-time
}

\author{
A.V. Minkevich \\ Department of Theoretical Physics and Astrophysics, Belarusian State University, Minsk \\ Belarus \\ Department of Physics and Computer Methods, Warmia and Mazury University in \\ Olsztyn, Olsztyn, Poland \\ E-mail: minkav@bsu.by, awm@matman.uwm.edu.pl
}

\begin{abstract}
The gravitational interaction at astrophysical scale is discussed within the framework of minimum gauge gravitation theory in Riemann-Cartan space-time. It is shown that the interaction of the vacuum torsion with the spin momenta of gravitating objects can lead into appearance of additional gravitational force in non-relativistic approximation, that can be manifested on astrophysical scale (galaxies, galactic clusters).
\end{abstract}

Keywords: gravitational interaction, vacuum torsion, spin moment of momentum

\section{Introduction}

The application of gauge invariance principle, which underlies the modern theory of fundamental physical interactions, by construction of gravitation theory leads to generalization of metric gravitation theory. Gauge gravitation theory in 4-dimensional Riemann-Cartan space-time $U_{4}$ (GTRC) which is known in literature as Poincaré gauge theory of gravity is the necessary generalization of metric gravitation theory by including the Lorentz group into the gauge group corresponding to gravitational interaction. After the appearance of the pioneer works [1-3], which initiated the development of GTRC, many scientists were engaged in the research of this theory, which is currently one of the important directions in the modern theory of gravitation $\ddagger$ Gravitational equations of GTRC and their physical consequences depend on the choice of gravitational Lagrangian $\mathcal{L}_{\mathrm{g}}$ as function of gravitational field strengths - the curvature $F^{i k}{ }_{\mu \nu}$ and torsion $S^{i}{ }_{\mu \nu}$ tensors, and also on $\ddagger$ See bibliography on gauge gravity theory in the monograph [6], including more than 3 thousand publications. 
the coupling of matter with gravitational field $\S$. By using minimal coupling the energymomentum and spin momentum tensors of gravitating matter play the role of sources of gravitational field. In the frame of gauge approach the Lagrangian of gauge field usually is given as function quadratic in the gauge field strength; existence of many invariants, that are quadratic in the curvature and torsion tensors, is notable feature of GTRC, moreover there is linear in the curvature invariant - scalar curvature $\|$. Because the detailed form of gravitational Lagrangian is unknown, GTRC based on the sufficiently general expression of $\mathcal{L}_{\mathrm{g}}$, including both the scalar curvature and various invariants quadratic in the curvature and torsion tensors with indefinite parameters, was investigated (see e.g. [7-16]). Restrictions on indefinite parameters of $\mathcal{L}_{\mathrm{g}}$ can be found in the request, that satisfying the correspondence principle with general relativity theory (GR), GTRC allows to solve some principal problems of GR and physical consequences of this theory are the most satisfactory. Some such restrictions were found from analysis of isotropic cosmology, built in the frame of GTRC (see $[10,11,13]$ and Refs herein). It was shown, that gravitational interaction, in the case of usual gravitating matter with positive values of energy density and pressure, differs from the one that is given in the frame of GR, at extreme conditions (extremely high energy densities and pressures), where limiting (i.e. maximum allowable) energy density appears [11], and also when energy density is very small (comparable with average energy density in the Universe at present epoch) and the vacuum gravitational repulsion effect is essential. This effect appears, because the physical space-time in the vacuum has the structure of Riemann-Cartan continuum with de Sitter metric, but not Minkowski space-time [10]. As result, the solution of the problem of cosmological singularity and the dark energy problem was obtained in the frame of GTRC.

This article is devoted to study gravitational interaction in astrophysics in the case of various objects, for which energy density is much smaller than limiting energy density. In general case the description of such systems in the frame of GTRC is difficult problem because of complexity of gravitational equations. The situation was simplifying, when minimum GTRC was determined [14]. Gravitational equations of minimum GTRC include three indefinite parameters, entered earlier in the frame of isotropic cosmology (see below) and in the case of spinless gravitating systems with energy densities, which are much smaller than limiting energy density, lead to gravitation equations for metric in the form of Einstein gravitation equations with effective cosmological constant [14]:

$$
G_{\lambda}^{\mu}=-\frac{1}{2 b}\left[T_{\lambda}^{\mu}+\delta_{\lambda}^{\mu} \frac{\left(1-\frac{b}{f_{0}}\right)^{2}}{4 \alpha}\right]
$$

$\S$ The definitions and notations of our previous work (see e.g. [9]) are used below. With the purpose to make quantitative estimations the light velocity $c$ is conserved in formulas.

I| Pioneer works dedicated to GTRC were connected with investigation of Einstein-Cartan theory, gravitational Lagrangian of which is given in the form of scalar curvature of $U_{4}$ [1-5]. 
where $G_{\lambda}^{\mu}$ is Einstein tensor. The correspondence principle with GR is fulfilled, if the following restriction for indefinite parameter $b$ is valid: $0<1-\frac{b}{f_{0}} \ll 1$, where $f_{0}=\frac{c^{4}}{16 \pi G}(G$ is Newton's gravitational constant) and the value of parameter $\alpha^{-1}$ corresponds to some high energy density, by which effective cosmological constant in eq. (1) corresponds to observable acceleration of cosmological expansion. The third small dimensionless indefinite parameter $\omega(0<\omega \ll 1)$ is absent in equations for discussed systems $\boldsymbol{\top}$.

By neglecting spin effects Newton's law of gravitational attraction is valid in nonrelativistic approximation and the description of various astrophysical objects in the frame of minimum GTRC practically coincides with that in GR, because the influence of cosmological constant in (1) is negligibly small at astrophysical scale. If we take into account that various astrophysical objects (stars in galaxies, galaxies in galactic clusters) possess the own moments of momentum, the question about the influence of these moments on dynamics of astrophysical objects appears because of the interaction of own moments of momentum considering as spin momentum with space-time torsion. It should be noted that Pioneer works connected with GTRC were dedicated to investigation of the role of spin momentum in gravitation theory [1-5]. Because in the frame of GTRC physical space-time in the vacuum has the structure of Riemann-Cartan continuum [10], in the case of the movement of rotating astrophysical object in Newtonian gravitational field spin effects can be manifested as a result of interaction of vacuum torsion with spin momentum of this object. The present paper is devoted to investigation of this problem. With this purpose the origin of the vacuum torsion and the dynamics of rotating particle in Riemann-Cartan space-time are discussed below.

\section{Isotropic cosmology in Riemann-Cartan space-time and vacuum torsion}

With the aim of studying the influence of vacuum torsion on gravitational interaction in astrophysics lets consider in more detail the definition of the vacuum torsion. First of all, note, that in the frame of classical field theory the geometric structure of physical space-time in the vacuum can be found by supposing that it does not change with the time and 3-space is homogeneous and isotropic. Then the vacuum torsion as characteristics of physical spacetime in the vacuum can be introduced on the base of equations of isotropic cosmology, built in the frame of GTRC [10]. Any homogeneous isotropic model (HIM) in Riemann-Cartan space-time is described by three functions of time: the scale factor of Robertson-Walker metric $R(t)$ and two torsion functions - scalar function $S_{1}(t)$ and pseudoscalar function $S_{2}(t)$. Cosmological equations generalizing Friedmann cosmological equations of GR take

I We don't discuss here systems under extreme conditions near the limiting energy density, where terms with the parameter $\omega$ in the gravitational equations play a fundamental role. 
the form [11]

$$
\begin{aligned}
& \frac{k}{R^{2}}+\left(H-2 S_{1}\right)^{2}-S_{2}^{2}= \\
& \frac{1}{6 f_{0} Z}\left[\rho c^{2}-6 b S_{2}^{2}+\frac{\alpha}{4}\left(\rho c^{2}-3 p-12 b S_{2}^{2}\right)^{2}\right], \\
& \dot{H}-2 \dot{S}_{1}+H\left(H-2 S_{1}\right)= \\
& -\frac{1}{12 f_{0} Z}\left[\rho c^{2}+3 p-\frac{\alpha}{2}\left(\rho c^{2}-3 p-12 b S_{2}^{2}\right)^{2}\right],
\end{aligned}
$$

where $H=\dot{R} / R$ (a dot denotes the differentiation with respect to $x^{0}=c t$ ), $k=+1,0,-1$ for closed, flat and open models respectively, $\rho$ is mass density, $p$ is pressure and $Z=$ $1+\alpha\left(\rho c^{2}-3 p-12 b S_{2}^{2}\right)$. The torsion functions $S_{1}$ and $S_{2}$ are:

$$
\begin{aligned}
& S_{1}=-\frac{\alpha}{4 Z}\left[\dot{\rho} c^{2}-3 \dot{p}+12 f_{0} \omega H S_{2}^{2}-12\left(2 b-\omega f_{0}\right) S_{2} \dot{S}_{2}\right], \\
& S_{2}^{2}=\frac{\rho c^{2}-3 p}{12 b}+\frac{1-\left(b / 2 f_{0}\right)(1+\sqrt{X})}{12 b \alpha(1-\omega / 4)}
\end{aligned}
$$

where

$$
X=1+\omega\left(f_{0}^{2} / b^{2}\right)\left[1-\left(b / f_{0}\right)-2(1-\omega / 4) \alpha\left(\rho c^{2}+3 p\right)\right] \geq 0 .
$$

Supposing that physical space-time in the vacuum is homogeneous and isotropic and does not change in time, we can obtain its structure from equations for HIM (2)-(6), directing the mass density and pressure and time derivatives to the zero. Such procedure is possible in the case of flat HIM $(k=0)$. Unlike GR (without cosmological constant), where according to Friedmann cosmological equations space-time in the vacuum is Minkowski space-time $(H=0)$, in the frame of GTRC space-time in the vacuum has the structure of RiemannCartan continuum with de Sitter metric [10]. It is connected with the presence of constant term - vacuum torsion - in expression (5) for $S_{2}^{2}$ :

$$
S_{2}^{2(v a c)}=\frac{1-\frac{b}{2 f_{0}}\left[1+\left(1+\omega\left(1-b / f_{0}\right) \frac{f_{0}^{2}}{b^{2}}\right)^{1 / 2}\right]}{12 \alpha b(1-\omega / 4)} .
$$

Then in accordance with eqs. (2)-(5) the vacuum value of $H^{2}$ (in the case $k=0$ ) is:

$$
H^{2(v a c)}=\frac{6 b^{2}}{f_{0}} \alpha S_{2}^{4(v a c)}\left[1-6 \alpha\left(2 b+\omega f_{0}\right) S_{2}^{2(v a c)}\right]^{-1},
$$

and $S_{1}^{(v a c)}=-\frac{3 f_{0} \alpha \omega H^{(v a c)} S_{2}^{2(v a c)}}{1-12 \alpha b S_{2}^{2(v a c)}}$. In asymptotics, when energy density is small $\left(\alpha \rho c^{2} \ll 1\right)$, by using the restriction $0<x=1-\frac{b}{f_{0}} \ll 1$ the expression (5) for $S_{2}^{2}$ in the lowest approximation with respect to $x$ takes the form:

$$
S_{2}^{2}=\frac{1}{12 b}\left[\rho c^{2}-3 p+\frac{1-b / f_{0}}{\alpha}\right]
$$


and as a result cosmological equations (2)-(3) in asymptotics take the form of Friedmann cosmological equations with effective cosmological constant:

$$
\begin{aligned}
& \frac{k}{R^{2}}+H^{2}=\frac{1}{6 b}\left[\rho c^{2}+\frac{1}{4 \alpha}\left(1-\frac{b}{f_{0}}\right)^{2}\right], \\
& \dot{H}+H^{2}=-\frac{1}{12 b}\left[\left(\rho c^{2}+3 p\right)-\frac{1}{2 \alpha}\left(1-\frac{b}{f_{0}}\right)^{2}\right] .
\end{aligned}
$$

According to (9)-(10) and in compliance with (7)-(8) we have:

$$
S_{2}^{2(v a c)}=\frac{1-b / f_{0}}{12 b \alpha}, \quad H^{2(v a c)}=\frac{\left(1-\frac{b}{f_{0}}\right)^{2}}{24 b \alpha} .
$$

The effective cosmological constant in (10)-(11) is induced by the vacuum torsion $S_{2}^{2(v a c)}$. Unlike standard $\Lambda C D M$-model effective cosmological constant appears in (10)-(11) as a result of solution of gravitational equations for HIM, that leads to the change of gravitational interaction, when energy density is small and comparable with cosmological constant - the vacuum gravitational repulsion effect, which leads to accelerating cosmological expansion at present epoch.

It should be noted, that the vacuum torsion at the beginning of cosmological expansion near limiting energy density, determined from equality $X=0$, leads to appearance of negative effective cosmological term in expression for the Hubble parameter. In the case of HIM filled with gravitating matter with equation of state $p=p(\rho)$, the Hubble parameter with its time derivative near a bounce in the first approximation with respect to $\sqrt{X}$ are:

$$
\begin{aligned}
& H_{ \pm}= \pm \frac{2 b^{2}}{3 f_{0}^{2} \omega \alpha} \sqrt{X}\left[(1 / 4 b)\left(\rho_{m} c^{2}+p_{m}\right)-\left(k / R^{2}\right)\right. \\
& \left.-\frac{1-b /\left(2 f_{0}\right)}{24 f_{0} \alpha}\right]^{1 / 2}\left[\left(3 \frac{1}{c^{2}} \frac{d p_{m}}{d \rho_{m}}+1\right)\left(\rho_{m} c^{2}+p_{m}\right)\right]^{-1} \\
& \dot{H}=\frac{4 b^{2}}{3 f_{0}^{2} \omega \alpha} \frac{(1 / 4 b)\left(\rho_{m} c^{2}+p_{m}\right)-\left(k / R^{2}\right)-\frac{1-b /\left(2 f_{0}\right)}{24 f_{0} \alpha}}{\left(3 \frac{1}{c^{2}} \frac{d p_{m}}{d \rho_{m}}+1\right)\left(\rho_{m} c^{2}+p_{m}\right)} .
\end{aligned}
$$

$H_{-}$- and $H_{+}$-solutions describe the stages of compression and expansion correspondingly. The constant term $\left(-\frac{1-b /\left(2 f_{0}\right)}{24 f_{0} \alpha}\right)$ in $(13)$ can be interpreted as a result of the influence of vacuum under extreme conditions, however, quantitatively this influence is not essential, because energy density near a bounce is close to limiting energy density $\left(\rho_{\max } c^{2}\right) \sim(\omega \alpha)^{-1}$.

Because of restriction $0<1-\frac{b}{f_{0}} \ll 1$ the vacuum value of $H$ and the vacuum torsion function $\left|S_{1}\right|$ are negligibly small in comparison with $\left|S_{2}^{(v a c)}\right|$. Owing to this, the curvature tensor (see $[12,9])$ has the following vacuum components:

$$
F_{12}^{12}=F_{13}^{13}=F_{23}^{23}=-S_{2}^{2(v a c)},
$$


and as will be shown below, the vacuum torsion $S_{2}^{(v a c)}$ can be important in astrophysics in non-relativistic approximation.

\section{Vacuum torsion and gravitational interaction at astrophysical scale}

With the purpose to study movement of an object with spin momentum in gravitational field, we will use the equations of motion for particle with orientation in Riemann-Cartan space-time $U_{4}$ obtained in [17] ${ }^{+}$. The particle orientation in such model discussed in [21] is specified by the related triad of orthonormal vectors $e_{(i)}^{\mu}(\mathrm{i}=1,2,3)$, which are purely spatial in the rest frame of the particle and orthogonal to the 4 -velocity vector $v^{\mu}$. The definition of relativistic angular velocity tensor $\Omega^{\mu \nu}$ in $U_{4}$ as function of vectors $e_{(i)}^{\mu}$ and $v^{\mu}$ was obtained as generalization of angular velocity tensor in Riemannian space-time given in [21] by replacing riemannian absolute derivatives with respect to proper time $\tau$ by absolute derivatives defined by means of total connection of $U_{4}$ that corresponds to the principle of minimal interaction with gravitational field. As result angular velocity tensor $\Omega^{\mu \nu}$ in $U_{4}$ was defined in the form $\Omega^{\mu \nu}=e_{(i)}^{\mu} \frac{D e_{(i)}^{\nu}}{d \tau}-\frac{1}{c^{2}} v^{\nu} \frac{D v^{\mu}}{d \tau}$, where $\frac{D}{d \tau}$ denotes absolute derivative determined by full connection of $U_{4}$. It should be noted that such definition of $\Omega^{\mu \nu}$ is consistent with the proper relativistic generalization of translational motion given in ref. [21].

In the simplest case of rotating particle interacting with a gravitational field in spacetime $U_{4}$, being described by relativistic Lagrange function $L=-m_{0} c^{2}+\frac{I}{4} \Omega_{\mu \nu} \Omega^{\mu \nu}\left(m_{0}\right.$ is the rest mass in the absence of rotation, and the constant $I$ has the meaning of the inertia moment) the Lagrange equations of translational and rotational motion take the following form * :

$$
\begin{aligned}
& \frac{D P_{\mu}}{d \tau}=\frac{1}{2} I \Omega_{\alpha \beta} F^{\alpha \beta}{ }_{\mu \lambda} v^{\lambda}+2 S^{\beta}{ }_{\alpha \mu} v^{\alpha} P_{\beta}, \\
& \frac{D \Omega_{\mu \nu}}{d \tau}=\frac{2}{c^{2}} \Omega_{\alpha[\nu} v_{\mu]} \frac{D v^{\alpha}}{d \tau},
\end{aligned}
$$

where generalized moment $P_{\mu}=m v_{\mu}-\frac{I}{c^{2}} v_{\alpha} \frac{D \Omega_{\mu}{ }^{\alpha}}{d \tau}\left(m=m_{0}+\frac{I}{4 c^{2}} \Omega_{\mu \nu} \Omega^{\mu \nu}=\right.$ const is rest mass of rotating particle) and generalized moment of momentum $j_{\mu \nu}=I \Omega_{\mu \nu}$ is identical to spin momentum of particle [17, 20]. Equations of motion (15) are a generalization of Papapetrou's equations for rotating particle in GR [22] for space-time $U_{4}$. As it is shown below the application of equations (15) can lead to important physical results in astrophysics in non-relativistic approximation.

By taking into account that in non-relativistic approximation $P_{i}=m v_{i}(\mathrm{i}=1,2,3)$, the influence of curvature tensor in the right side of translational equations (15) can become apparent by means of vacuum curvature (14), and also the formulas for angular velocity

+ Dynamics of spinning matter in $U_{4}$ was investigated in a number of works (see e.g. [4], [18], [19], [20]).

* In [17] the curvature tensor was defined with opposite sign and the signature $(+2)$ was used. 
vector $\Omega_{i}=\frac{1}{2} \epsilon_{i k l} \Omega^{k l}\left(\epsilon_{i k l}\right.$ is Levi-Chivita symbol) and $g_{00}=1+\frac{2 \phi}{c^{2}}$ ( $\phi$ is newtonian potential), we can transform equations (15) to the following form (rectangular cartesian coordinate system is used) :

$$
\begin{aligned}
& m \frac{d \mathbf{v}}{d t}=-m \frac{d \phi}{d \mathbf{r}}-I S_{2}^{2(v a c)}[\mathbf{\Omega}, \mathbf{v}], \\
& \frac{d \boldsymbol{\Omega}}{d t}=-S_{2}^{(v a c)}[\boldsymbol{\Omega}, \mathbf{v}] .
\end{aligned}
$$

Besides Newtonian force of gravitational attraction $\mathbf{F}_{\mathbf{N}}=-m \frac{d \phi}{d \mathbf{r}}$, the right side of equation of translational motion (16) includes additional gravitational force connected with interaction of vacuum torsion with spin momentum of particle $\mathbf{F}_{\mathbf{v}}=-I S_{2}^{2(v a c)}[\boldsymbol{\Omega}, \mathbf{v}]$. This force depends on vacuum torsion $S_{2}^{(v a c)}$, for value of which the following estimation in dependence on indefinite parameter $x=1-\frac{b}{f_{0}}$ can be obtained. By taking into account that the average mass density in the Universe at present epoch according to (10) is of order $\rho_{1}=\frac{x^{2}}{4 c^{2} \alpha} \sim 10^{-26} \frac{\mathrm{kg}}{\mathrm{m}^{3}}$, we obtain that at the first approximation with respect to $x$ :

$$
S_{2}^{2(v a c)}=\frac{16 \pi G}{3 c^{2} x} \rho_{1} \sim \frac{1,2 \cdot 10^{-52}}{x}\left(\mathrm{~m}^{-2}\right) .
$$

This estimation gives certain restrictions for $S_{2}^{(v a c)}$ because $x \ll 1$.

As example of application (16), we will consider the motion of rotating particle in spherically symmetric Newtonian gravitational field created by mass $M$. In the case of circular motion in plane $X O Y$ (centrum of mass $M$ is in origin of coordinates, vector of orbital moment is directed along the axe $O Z$ ) the additional gravitational force is $\mathbf{F}_{\mathbf{v}}=I \Omega_{3} S_{2}^{2(v a c)} v \frac{\mathbf{r}}{r}$ and its character depends on relative orientation of spin and orbital moments. We have the force of attraction or repulsion, depending on $\Omega_{3}<0$ or $\Omega_{3}>0$, as result the value of gravitational force in (16) is $F=G \frac{m M}{r^{2}} \pm I\left|\Omega_{3}\right| S_{2}^{2(v a c)} v$. By taking into account that this force is centripetal, we obtain the following dependence of velocity on distance from centrum and parameters of particle and gravitational field:

$$
v= \pm \frac{I}{2 m}\left|\Omega_{3}\right| S_{2}^{2(v a c)} r+\left[\left(\frac{I}{2 m}\left|\Omega_{3}\right| S_{2}^{2(v a c)} r\right)^{2}+\frac{G M}{r}\right]^{\frac{1}{2}} .
$$

The formula (18) is approximative and can be applied for limited time intervals because the angular velocity vector changes according to rotational motion equation (16) that leads to the change of trajectory plane.

Taking into account restriction (17), we will consider, for which astrophysical objects the detected interaction with vacuum torsion can manifest itself. First of all, we can get a torsion constraint by applying the obtained results to the planets of the solar system, where this interaction does not manifest itself. Using the parameters of the Earth $\left(m_{E} \sim 3 \cdot 10^{-6} M_{\odot} \sim\right.$ $6 \cdot 10^{24} \mathrm{~kg} ; r \sim 1,5 \cdot 10^{8} \mathrm{~m} ; v \sim 3 \cdot 10^{4} \mathrm{~m} / \mathrm{s} ; \Omega \sim 7,3 \cdot 10^{-5} \mathrm{~s}^{-1}$; angle between orbital and spin moments is $23,45^{\circ}$ ), we find that the ratio of additional gravitational force and Newtonian 
force is $F_{v} / F_{N} \sim 0,56 \cdot 10^{16} S_{2}^{2(v a c)}$, that leads to a restriction $S_{2}^{2(v a c)} \ll 10^{-16}\left(\mathrm{~m}^{-2}\right)$. Another restriction for the vacuum torsion we can obtain by supposing that relative change of Earth's own angular velocity per one period of movement around the Sun is small; in accordance with rotation equation of motion (16) we obtain $\frac{|\Delta \Omega|}{\Omega} \sim 0,4 \cdot 10^{14}\left|S_{2}^{(v a c)}\right|$; it follows from $\frac{|\Delta \Omega|}{\Omega} \ll 1$ that $\left|S_{2}^{(\text {vac })}\right| \ll 10^{-14}\left(\mathrm{~m}^{-1}\right)$ and hence $S_{2}^{2(\text { vac })} \ll 10^{-28}\left(\mathrm{~m}^{-2}\right)$. Similar estimation can be obtained by considering other planets of the solar system.

Now we will consider what possible role the vacuum torsion can play at astrophysical scale - galaxies and galactic clusters. By taking $S_{2}^{2(v a c)} \sim 10^{-30}\left(\mathrm{~m}^{-2}\right)$, we will use formula (18) in the case of attraction force to the star similar to Solar $\left(I / m \sim 10^{18} \mathrm{~m}^{2},\left|\Omega_{3}\right| \sim 10^{-6} \mathrm{~s}^{-1}\right)$ moving in galaxy similar to Andromeda $\left(M=2 \cdot 10^{41} \mathrm{~kg}\right)$. As numerical analysis shows, at distances $r \sim 10 \mathrm{kpc}\left(1 \mathrm{kpc}=0,3086 \cdot 10^{20} \mathrm{~m}\right)$ Newtonian term in formula (18) plays the definitive role, and terms, connected with additive gravitational force, give corrections of approximately 0,001 part of the Newtonian force. However, by further increase of $r$ essential growth of velocity $v$ takes place according to (18) and by sufficiently large distances the influence of additional gravitational force can far exceed the effects associated with the Newtonian force; this effect can be essential in galactic clusters, where we deal with vast space scale of order $10 \mathrm{Mpc}$ and more.

Although, given above consideration was made in the frame of minimum GTRC, similar effects take place in other GTRC, because of existence of the vacuum torsion. In addition to the influence of vacuum torsion in astrophysics, discussed above, the minimum GTRC can lead to the appearance of torsion associated with the angular (spin) moments of astrophysical objects. If the average value of the angular moment is not negligible for some area of space, gravitational equations lead to the appearance of additional torsion associated with these moments. Then the equations for the metric differ in form from the equations (1), acquiring certain corrections. The study of the corresponding phenomena involves setting the initial distribution for gravitating matter with angular moments using a certain model for the spinning matter. The appearance of an additional gravitational force (in addition to the Newtonian force of gravitational attraction) in the non-relativistic approximation makes these studies more interesting from physical point of view in connection with the dark matter problem.

Currently, there are a number of works performed to solve the problem of dark matter in the framework of theories of gravitation in pseudo-Riemannian space-time $f(R)$, as well as in the framework of theories of gravitation in teleparallelism space-time $f(T)$ (see $[24,25,26]$ and Refs there). The solution of the problem in these works is connected with the search for the modified Newtonian potential that provides an appropriate modification of the gravitational force in astrophysics. From a physical point of view, as part of our consideration, the reason for the modification of the gravitational force in astrophysics is different, namely, it is associated with the appearance of the force caused by the interaction 
of torsion with the spin moments of astrophysical objects in addition to the Newtonian gravitational force. Within the framework of GTRC, the nature of gravitational interaction differs significantly from what we have in $f(R)$ and $f(T)$ theories, and it is determined both by curvature and torsion together. At the same time within GTRC the nature of torsion, which along with metric, both are independent gravitational characteristics from each other, differs in principle from that in the frame of $f(T)$ theories of gravity, where the torsion and metric are defined as functions of tetrad coefficients. In contrast to the GTRC, whose gauge group includes the Lorentz group in addition to the translations group, the $f(R)$-theory and the $f(T)$-theory can be introduced in the framework of the gauge approach when considering the 4-translations group as a gauge group $\sharp$.

It should be noted that discussed phenomena connected with the change of gravitational interaction have essentially non-linear origin. Because of non-linear character of gravitating vacuum, approximative analysis of GTRC based on investigation of linearized theory and perturbations of gravitational field above Minkowski space-time [8] has approximative character. In particular, this concerns the analysis of particle content of GTRC, where it would be taken into account not only deviation of space-time metric in the vacuum from that of Minkowski space-time, but also the presence of vacuum torsion (compare with [23]). It should be noted that the deviation of the structure of the vacuum space-time in the frame of GTRC from Minkowski space-time, which is essential at cosmological and astrophysical scale, can be unimportant by local analysis given in [8] because of smallness of corresponding characteristics of metric and torsion for the vacuum. However, we have to consider corresponding results of [8] as approximative, whose range of applicability is limited by weak fields.

\section{Conclusion}

Research of gravitation theory in Riemann-Cartan space-time shows that satisfying the correspondence principle with general relativity theory GTRC leads to fundamental differences, associated with the gravitational interaction not only in cosmological but also in astrophysical scales. Distinctions are connected with geometrical structure of physical spacetime, namely with space-time torsion. Unlike isotropic cosmology, where space-time torsion is created by spinless matter and spin momentum of gravitating matter is not demonstrated, the interaction of torsion with spinning matter can play principal role in astrophysics. As it is shown in this paper, the interaction of the vacuum torsion with spin momentum of astrophysical objects leads to corrections of gravitational interaction, namely to appearance of additional gravitational force, which can have an influence on their movement. The search of possible observational demonstrations of this phenomenon is of direct physical interest.

$\sharp$ Discussion of these issues is available in [16]. 


\section{Acknowledgments}

The work is partially supported by the Belarusian National Research Program 'Convergence2020'. The author is grateful to the participants of the Seminar of the Department of Theoretical Physics and Astrophysics of the Belarusian State University for the discussions on the content of this article. I am thankful to my wife Eleonora for her permanent support.

\section{References}

[1] Kibble T W B 1961 J. Math. Phys. 2212.

[2] Brodskii A M, Ivanenko D and Sokolik G A 1961 JETP 41, 1307 (in Russian); 1962 Acta Phys.Hungar. $14,21$.

[3] Sciama D W, In: Recent Developments in General Relativity, Festschrift for Infeld (Pergamon Press, Oxford; PWN, Warsaw, 1962), p. 415.

[4] Trautman A 1973 Bull. Acad. Pol. Sci., Ser. sci. math., astr., phys. 21, 345; In: Encyclopedia of Mathematical Physics 2, J.-P. Francoise et al. (eds.), Elsevier, Oxford (2006), p. 189.

[5] Hehl F W, von der Heyde P, Kerlik G D and Nester J M 1976 Rev. Mod. Phys. 48, 393.

[6] Vladimir N. Ponomarev, Andrei O. Barvinsky, Yuri N. Obukhov, 2017 Gauge Approach and Quantization Methods in Gravity Theory, Moscow, Nauka, p. 201-345.

[7] Minkevich A V 1980 Vestsi Akad. Navuk BSSR, ser. fiz.-mat. navuk, No 2, 87; 1980 Phys. Lett. A 80, 232.

[8] Hayashi K and Shirafuji T 1980 Progr. Theor. Phys. 64, 866; 883; 1435; 2222.

[9] Minkevich A V, Garkun A S and Kudin V I 2013 JCAP 03, 40.

[10] Minkevich A V 2011 Mod. Phys. Lett. A 26, 259.

[11] Minkevich A V 2011 JETP Lett. 94, 831.

[12] Minkevich A V, Garkun A S and Kudin V I 2007 Class. Quantum Grav. 24, 5835.

[13] Minkevich A V 2016 Grav. Cosmol. 22, 148.

[14] Minkevich A V 2017 Grav. Cosmol. 23, 311.

[15] Minkevich A V 2016 Int. J. Mod. Phys. A 31, 1641011.

[16] Minkevich A V, In: The Fourteenth Marcel Grossmann Meeting on Recent Developments in Theoretical and Experimental General Relativity, Astrophysics and Relativistic Field Theories (World Scientific Publishing Company, 2017, in 4 Volumes, Editors: Massimo Bianchi, Robert T. Jantzen and Remo Ruffini) p.1156-1166.

[17] Minkevich A V, Sokolsky A A 1975 Vestsi Akad. Navuk BSSR, ser. fiz.-mat. No 4, 72 (in Russian).

[18] Krechet V G, Ponomarev V N 1976, In: Problems of gravitation theory and elementary particles, Moscow, Atomizdat, 7, p. 174-183 (in Russian).

[19] Collish H 1975 Die Bewegung von Probeteilchen in einem Raum-Zeit Continuum mit Torsion, Clausthal: ed. "Techn. Univ.", 84 p.

[20] Minkevich A V, Karakura F 1983 J. Phys. A: Math. Gen., 16, 1409.

[21] Schild A, Schlosser J A 1965 J. Math. Phys. 6, 1299.

[22] Papapetrou A 1951Proc. Roy. Soc. A, 209, 248.

[23] Nair V P, Randjbar-Daemi S, Rubakov V 2009 Phys. Rev. D 80, 104031.

[24] Capozziello S, Janović P, Borka Janović V and Borka D, 2017 JCAP 06, 044.

[25] Andrew Finch and Jackson Levi Said, 2018 Eur. Phys. J. C78, 560.

[26] Jamil M, Momeni D, Myrzakulov R 2012 Eur. Phys. J. C72, 2122. 